\title{
The merger stage of the ultra-luminous infrared galaxy IRAS 03158+4227
}

\author{
H. Meusinger ${ }^{1, \star}$, B. Stecklum ${ }^{1, \star}$, C. Theis ${ }^{2}$, and J. Brunzendorf ${ }^{1, \star}$ \\ 1 Thüringer Landessternwarte Tautenburg, 07778 Tautenburg, Germany \\ 2 Institut für Theoretische Physik und Astrophysik der Universität Kiel, Olshausenstr. 40, 24098 Kiel, Germany
}

Received 12 July 2001 / Accepted 24 September 2001

\begin{abstract}
We examine the merger stage of IRAS 03158+4227, one of the most luminous ULIRGs from the IRAS 2 Jy sample. Deep optical images are presented along with high-resolution NIR images and optical lowresolution spectra. IRAS $03158+4227$ is confirmed as a component of an equal-luminosity binary galaxy with a nuclei separation of $47 h_{75}^{-1} \mathrm{kpc}$. A long lopsided tail emanating from the companion, which harbours an active nucleus, is the most prominent peculiar feature of the binary. The results of numerical simulations permit the interpretation of this structure as a product of the tidal interaction between the two components. If the infraredactivity of IRAS $03158+4227$ is also dynamically triggered by this process, this would imply that the ULIRG phenomenon is not restricted to the final stage of a binary merger. Alternatively, the system may be a multiple merger where the partner(s) has/have already coalesced.
\end{abstract}

Key words. galaxies: interactions - galaxies: nuclei - galaxies: starbursts - infrared: galaxies

\section{Introduction}

The characteristics of galaxies that emit a substantial amount of their bolometric luminosity in the far infared (FIR) has been a matter of debate since their discovery (e.g., Rieke \& Low 1972; Joseph \& Wright 1985; Soifer et al. 1987). Particular interest is focussed on the class of ultra-luminous infrared galaxies (ULIRGs), i.e. galaxies with quasar-like infrared luminosities of $L_{\mathrm{IR}} \geq 10^{12} L_{\odot}$ (e.g., Sanders \& Mirabel 1996; Kennicutt 1996; Genzel et al. 1998; Rigopoulou et al. 1999; Scoville et al. 2000). Nuclear starbursts and/or AGN activity, dynamically triggered by gravitational interactions, are thought to be the energy sources in the cores of ULIRGs. Absorption and re-emission in the FIR is expected to be a consequence of thermalization of radiation by large masses of dust grains.

It has been demonstrated by a large number of studies that a high fraction of luminous and ultraluminous infrared galaxies show morphological peculiarities, such as tidal debris and double nuclei, indicative of gravitaional perturbations. For instance, all ULIRGs of the

Send offprint requests to: H. Meusinger,

e-mail: meus@tls-tautenburg.de

* Visiting Astronomer, German-Spanish Astronomical Centre, Calar Alto, operated by the Max-Planck-Institut for Astronomy, Heidelberg, jointly with the Spanish National Comission for Astronomy. original IRAS bright galaxy survey (BGS; Sanders et al. 1988a) show indications of strong gravitational interaction or merging. Published values for the interacting/merging fraction among the ULIRGs from various studies cover the range from $50 \%$ to about $100 \%$ (e.g., Aurière et al. 1996; Clements et al. 1996; Murphy et al. 1996; Duc et al. 1997; Borne et al. 2000). Murphy et al. have analysed near-infrared (NIR) and visual images for 46 luminous infrared galaxies with $L_{\mathrm{IR}}>8.5 \times 10^{11} L_{\odot}$ from the IRAS 2 Jy sample. After combining their sample with the BGS, they find that $95 \%$ of the galaxies in the combined sample show evidence for current or past interactions. There are only three ULIRGs in the Murphy et al. sample that do not, to the limits of the images given there, show indications of interactions. Among them is IRAS 03158+4227, one of the most luminous ULIRGs $\left(\log L_{\mathrm{IR}} / L_{\odot}=12.55, f_{60} / f_{100}=0.95\right)$. This seems astonishing since the fraction of perturbed systems among the FIR-bright galaxies is known to increase with $L_{\mathrm{IR}}$ (e.g., Sanders \& Mirabel 1996, and references therein).

On the numerical side, simulations clearly show that the major morphological features observed in many peculiar galaxies are explained as being due to tidal forces during galaxy encounters (e.g., Toomre \& Toomre 1972; Barnes \& Hernquist 1992; Bekki \& Noguchi 1994; Spoke 1997; Mihos et al. 1998). Self-consistent models of tidally disturbed galaxies indicate high gas concentrations 
in the centres due to strong and sudden gaseous inflow (Negroponte \& White 1983; Noguchi 1991; Barnes \& Hernquist 1991, 1996; Mihos \& Hernquist 1996). The models predict that merger-driven gas-dynamics and associated star formation may result in spectacular starbursts (e.g. Noguchi \& Ishibashi 1986; Mihos \& Hernquist 1996), although there is considerable uncertainty about the treatment of star formation and of the feedback from young stars in the simulations. Galaxy merger and the ULIRG phenomenon were tied together in the models by Mihos \& Hernquist (1996).

ULIRGs are widely claimed to represent the final stages of merging galaxies. Murphy et al. (1996) give projected linear separations of less than a few kpc for a large fraction of their sample. In the case of IRAS $03158+4227$, Murphy et al. noticed a nearby, resolved component which they described as "not apparently interacting with the primary galaxy". IRAS $03158+4227$ was therefore not included by these authors in their sample of double-nuclei systems and was subsequently considered a single system where the nuclei separation must be smaller than the available resolution of $0 . \prime 8$. The projected linear distance to the nucleus of the galaxy mentioned by Murphy et al. amounts to $18^{\prime \prime}$, corresponding to a projected linear separation of $47 \mathrm{kpc}$ (throughout this paper we adopt an Einsteinde Sitter comology with $H_{0}=75 \mathrm{~km} \mathrm{~s}^{-1} \mathrm{Mpc}^{-1}$ ). There are only a few 2 Jy ULIRGs with nuclear distances larger than $10 \mathrm{kpc}$ and only one other system (IRAS 14394+5332) with a separation of about $50 \mathrm{kpc}$.

In this paper, we present deep optical imaging, highresolution (adaptive optics) NIR imaging, and optical spectroscopy of the remarkable ULIRG IRAS03158+4227. The observational data (imaging and spectroscopy) are described in Sect. 2. The results are presented in Sect. 3 and are discussed in the context of simulations in Sect. 4. Finally, conclusions are given in Sect. 5 .

\section{Observations and data reduction}

Most of the observations presented here were taken at the German-Spanish Astronomical Centre on Calar Alto, Spain. A summary of relevant data for all observations is given in Table 1.

Deep direct optical imaging was performed with the focal reducer camera CAFOS at the $2.2 \mathrm{~m}$ telescope in the $B, R$, and $I$ band and without filters. CAFOS was equipped with a SITe CCD with a scale of $0.5 /$ pixel. The conditions were always photometric with a seeing typically of about $1^{\prime \prime}$. Unfortunately, IRAS $03158+4227$ is located only about $2^{\prime}$ away from the bright foreground star HD $20489(V=8.6)$ and 7 "! 4 from the USNO-2 star No. 275-02191303 ( $B=16.2, R=14.9)$. Therefore, we took several sets of relatively short exposures (typically between 100 and $300 \mathrm{~s}$ ) to avoid saturation effects of the CCD. The total integration time amounts to 2.8 hours. MIDAS standard algorithms were applied for the data reduction. The combined image was PSF-deconvolved
Table 1. Log of observations.

\begin{tabular}{rrrr}
\hline $\begin{array}{c}\text { filter/ } \\
\text { grism }\end{array}$ & $\begin{array}{r}t_{\exp } \\
{[\mathrm{s}]}\end{array}$ & instrument & epoch \\
\hline$J$ & 360 & ALFA/Omega-Cass & Sep. 98 \\
$H$ & 180 & ALFA/Omega-Cass & Sep. 98 \\
$K^{\prime}$ & 90 & ALFA/Omega-Cass & Sep. 98 \\
- & 700 & CAFOS & Jul. 98 \\
$B$ & 4560 & CAFOS & Jul. 98, 00 \\
$R$ & 3700 & CAFOS & Jul. 99, 00 \\
$I$ & 820 & CAFOS & Jul. 00 \\
$B 400$ & $1200(\mathrm{G} 1)$ & CAFOS & Jul. 99 \\
$B 400$ & $3000(\mathrm{G} 2)$ & CAFOS & Jul. 99 \\
$R$ & 1200 & TLS Schmidt & Jan. 99 \\
$I$ & 960 & TLS Schmidt & Dec. 99 \\
\hline
\end{tabular}

using the Lucy-Richardson method (MIDAS procedure deconvolve/flucy).

High-resolution imaging in the $J, H$, and $K^{\prime}$ bands was performed using the adaptive optics system ALFA in combination with the Omega-Cass camera (Hippler et al. 1998) at the $3.5 \mathrm{~m}$ Calar Alto telescope. Omega-Cass utilizes a $1024 \times 1024$ HAWAII detector and was operated at the pixel scale of $0 . \prime 08$. Since there is no sufficiently bright star close to IRAS $03158+4227$ which could be used for wavefront sensing and with the laser guide star being not operational at the time of the observations, we corrected the static aberrations of the telescope on a nearby star and imaged the target with the deformable mirror "frozen". Although this procedure does not yield diffraction-limited resolution, it improved the image quality considerably, leading to sub-arcsecond resolution. Two adjacent fields were observed for deriving the sky frames. During the data processing, the images were rebinned in order to enhance the signal-to-noise ratio which led to a final pixel scale (from the astrometric solution) of $0{ }^{\prime \prime} 155$. After correction for flat field and bad pixels, the images were filtered using the wavelet algorithm of Pantin \& Starck (1996) to minimize noise amplification in the subsequent RichardsonLucy deconvolution. The USNO-2 star No. 275-02191303 served as PSF reference for the deconvolution. $J H K^{\prime}$ photometry was derived from the non-deconvolved images and tied to the $J H K^{\prime}$ magnitudes of the PSF star according to its entry in the 2MASS Second Incremental Release Point Source Catalog (Cutrie et al. 2000). The derived fluxes (Fig. 2) refer to a synthetic aperture of 7 ". 75 diameter. The photometric error amounts to $0.06 \mathrm{mag}$. In order to assess the separation of a possible double nucleus, the FWHM of the images of the ULIRG in the three filters were compared to those of stars in the field. The average stellar $F W H M$ s derived from Gaussian fits amount to $0.600,0$. 57 , and 0.58 for $J, H$, and $K^{\prime}$, respectively. The $F W H M$ s of the ULIRG are $00^{\prime \prime} 74,0$.'70, and $0 . \prime 71$. This leads to beamdeconvolved sizes of 0 . 44,0 .'40, and 0 .' 40 for the angular extent of the emitting core region of the ULIRG. 
Low-resolution spectra of both IRAS $03158+4227$ and its nearest neighbour galaxy were taken with CAFOS equipped with the grism B 400 which is suitable for the wavelength range $\lambda=3200 \ldots 8000 \AA$. With a slit width of $1^{\prime \prime} 2$ the spectral resolution is about $20 \AA$.

Finally, we observed the field of IRAS $03158+4227$ with the Tautenburg Schmidt telescope at moderate seeing of about $2^{\prime \prime}$. The Schmidt camera was equipped with a $2 \mathrm{k} \times 2 \mathrm{k}$ SITe CCD with pixel size of $24 \mu \mathrm{m} \times$ $24 \mu \mathrm{m}$ which yields a field size of $40^{\prime} \times 40^{\prime}$. These images were used only to evaluate the large-scale environment of IRAS $03158+4227$.

\section{Results}

Figure 1 shows a moderately deep optical image of the field around IRAS $03158+4227$. The data for the IRAS error ellipse were taken from the IRAS Point Source Catalogue, the coordinates for the objects on the optical image are from the astrometric solution for the field of the Perseus cluster of galaxies (Brunzendorf \& Meusinger 1999). The IRAS error ellipse clearly overlaps with the optical image of a faint galaxy (G1) with $z=0.13$ (NASA Extragalactic Database, NED). At the distance of the galaxy, the size of the field shown in Fig. 1 corresponds to about $0.5 \mathrm{Mpc} \times$ $0.5 \mathrm{Mpc}$. There is no sign of a dense galaxy cluster or a rich group of galaxies around IRAS 0315+4227. Several small and faint galaxy images are seen in the field, but a substantial fraction may be dwarf galaxies of the Perseus cluster in the foreground.

Murphy et al. (1996) presented a $K$-band image taken with the Palomar 200 inch telescope at a seeing of 0.8 that shows neither evidence of a neighbour galaxy outshined by the brighter foreground star S1 on a less resolved image, nor for a double nucleus of G1. As mentioned in Sect. 1, IRAS 03158+4227 was assumed to be a late merger with nuclei separation of less than 0. ' 8 , even though it was noted by Murphy et al. (1996) that there is a galaxy (G2) at a distance of $18^{\prime \prime}$. The two galaxies G1 and G2 have approximately the same apparent magnitudes; after calibrating our $B$-band image using stars from the USNO-A2.0 Catalogue (Monet et al. 1998) we find $B=18.6$ and 18.4 for G1 and G2, respectively. This corresponds to $M_{B} \approx-21.4$ and -21.6 (for $A_{B}=0.8 \mathrm{mag}$ from the $\mathrm{NED}^{1}$, and a $k$-correction $k_{B}=0.5 \mathrm{mag}$ from Coleman et al. 1980) and to a very strong FIR excess of $\log L_{\mathrm{FIR}} / L_{B}=2.3$ for G1 (see e.g., Soifer et al. 1987 for the definitions of $L_{\mathrm{FIR}}$ and $\left.L_{B}\right)$.

The spectral energy distribution (SED) of IRAS $03158+4227$ is shown in Fig. 2. The SED is typical for ULIRGs, with the bulk of the luminosity radiated at farinfrared wavelengths. In addition to the data points shown in Fig. 2, we note that IRAS $03158+4227$ is identified with

1 The NASA/IPAC Extragalactic Database (NED) is operated by the Jet Propulsion Laboratory, California Institute of Technology, under contract with the National Aeronautics and Space Administration.

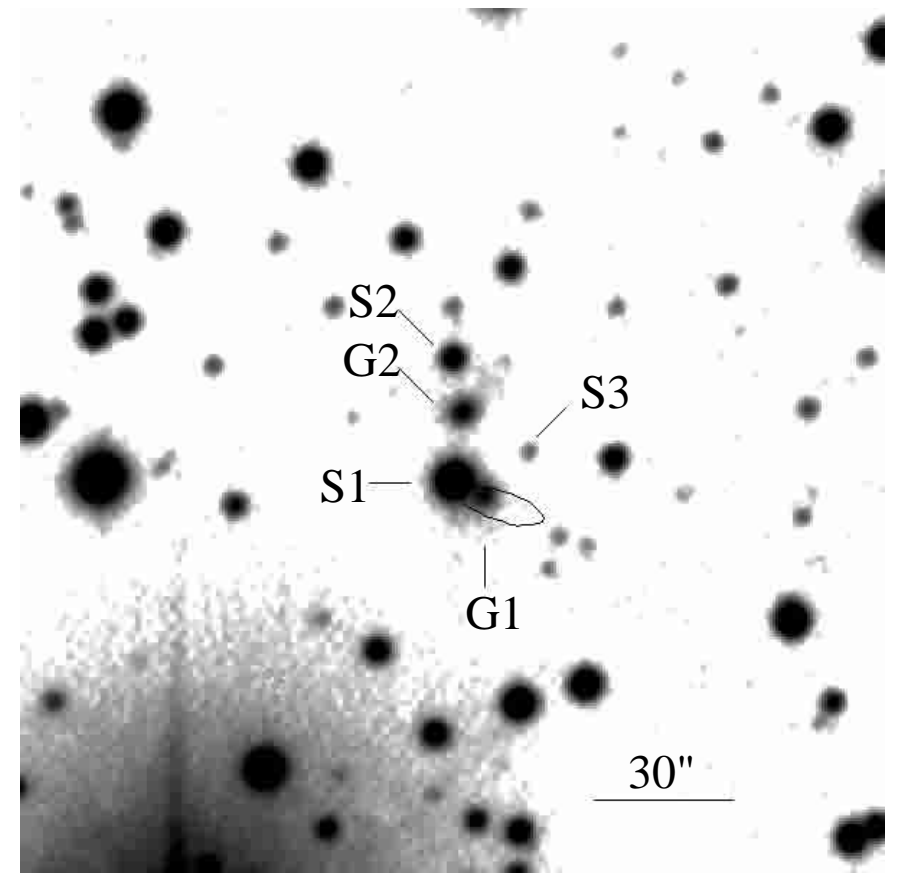

Fig. 1. IRAS error ellipse of IRAS 03158+4227. Coaddition of $R$ - and $I$-band images taken with the Tautenburg Schmidt camera. The size of the image is $200^{\prime \prime} \times 200^{\prime \prime}$; $\mathrm{N}$ is up, $\mathrm{E}$ is left. S1 to S3 designate stars, G1 and G2 indicate galaxies.

a radio continuum source in the NVSS (Condon et al. 1998). The flux density of $12.4 \mathrm{mJy}$ at $1.4 \mathrm{GHz}$ corresponds to a radio-to-infrared flux ratio (for definition see Helou et al. 1985) $q=2.64$. This is in agreement with the well-known correlation between the flux densities in the infrared and the radio continuum (see Sanders \& Mirabel 1996 , and references therein) where $\langle q\rangle \approx 2.35$ for most of the luminous infrared galaxies in the BGS, but is larger for the galaxies with highest infrared luminosities. (For instance, Helou et al. found $q=2.60$ for Arp 220.)

The optical images (Fig. 3) clearly reveal that G2 has a faint, but very extended material arm on the side opposite to G1. This feature is seen in all optical bands, and the Lucy-Richardson-deconvolved image shows that it is quite narrow, as expected for tidal tails. In the optical bands, the arm is about 3 mag fainter than the main body of G2. The surface brightness of the brightest part of the tail is estimated to be $\mu_{B} \approx 25 \mathrm{mag} \mathrm{arcsec}^{-2}$, the projected linear extent is about $70 \mathrm{kpc}$. Illustrative examples for similar tidal structures with one dominating arm can be found among the Arp (1966) and Arp \& Madore (1987) galaxies (e.g., Arp 107, 110, 129, 173, 252, 255, AM 2350-302, AM 0552-324, AM 0427-475). For the host galaxy of the ULIRG itself, tidal signatures are much weaker, although there appears to be some fuzz around G1. Several brighter spots may represent tidal debris, perhaps including huge star-forming regions; but clearly, none of these spots is bright enough to be considered a second nucleus of G1. Unfortunately, it can not be excluded that 


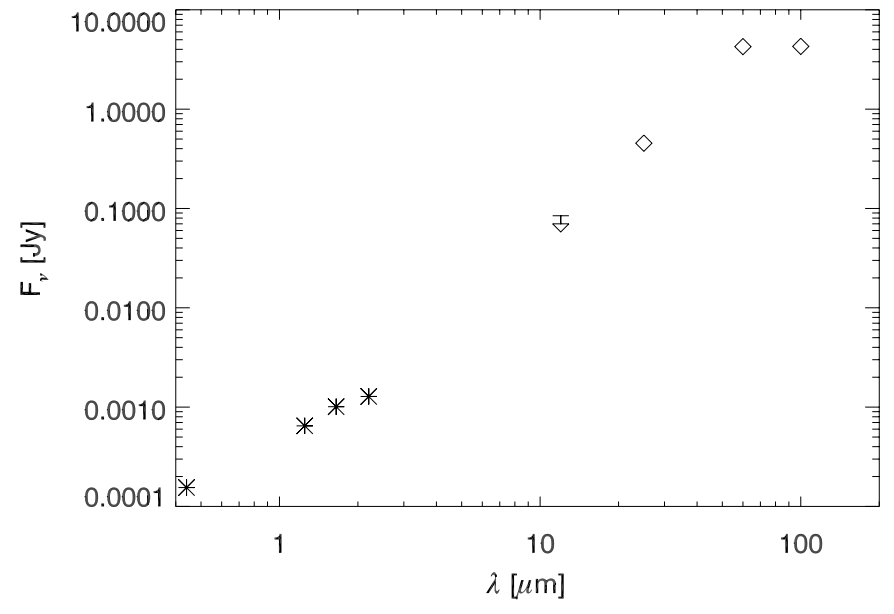

Fig. 2. Spectral energy distribution (SED) of IRAS 03158+4227. Asterisks denote our measurements while diamonds mark the IRAS fluxes. (The data point at $12 \mu \mathrm{m}$ represents an upper limit.)

faint morphological features of G1 are hidden behind the bright star S1.

The radial intensity profiles of G1 and G2 in the $R$-band are shown in Fig. 4. G1 may be approximated by two components with different scalelengths. Alternatively, it can be classified as an "E-like" ULIRG: the radial surface density profile is reasonably fit by a deVaucouleurs $r^{1 / 4}$-law over the range $R \approx 4 \ldots 30 \mathrm{kpc}$. (The innermost 1..5 were excluded from the analysis.) According to Sanders et al. (2000), about one third of the ULIRGs from the 1 Jy sample are classified as "E-like". Contrary to G1, the profile of G2 is better approximated by an exponential law which suggests a "disk-like" structure.

The images in the NIR-bands are shown in Fig. 5. These high-resolution images were taken to search for point sources indicating either a close double (or multiple) nucleus with small separations or a nucleus outshined by the bright star S1 in the optical image, rather than to evaluate extended structures of low-surface brightness. For such an aim, observations at longer wavelengths are needed, since the morphology of the central parts of ULIRGs is strongly affected by dust obscuration. As expected (cf. Sanders et al. 2000), the light distribution in $K^{\prime}$ is very compact for the galaxy G1 which appears as a point source. Figure 5 does not reveal any signs of a double nucleus in G1 or for a nucleus close to S1 down to the resolution (beam-deconvolved size) of 0.440 . Of course, there remains the possibility of an incidental superposition of the image of $\mathrm{S} 1$ and a further nucleus, but the probability for such a configuration is very low.

The optical spectra of G1 and G2 (Fig. 6) show strong emission lines; the equivalent widths $(E W \mathrm{~s})$ are listed in Table 2. A radial velocity difference between G1 and G2 of $240 \mathrm{~km} \mathrm{~s}^{-1}$ is estimated, but the uncertainty due to the low spectral resolution is quite large (about $\pm 200 \mathrm{~km} \mathrm{~s}^{-1}$ ).

IRAS 03158+4227 has sometimes been classified as a Seyfert 2, whereby strong absorption was invoked as the
Table 2. Equivalent widths $E W$ (in units of $\AA$ ) of the emission lines measured for the galaxies G1 and G2.

\begin{tabular}{lrr}
\hline & $\mathrm{G} 1$ & $\mathrm{G} 2$ \\
\hline$E W(\mathrm{H} \beta)$ & $3.5 \pm 0.4$ & $2 \pm 2$ \\
$E W([\mathrm{O} \mathrm{III}] 5007)$ & $8.0 \pm 0.4$ & $59 \pm 1$ \\
$E W([\mathrm{O} \mathrm{I}] 6300)$ & $8.0 \pm 0.4$ & $0.1 \pm 0.2$ \\
$E W(\mathrm{H} \alpha+[\mathrm{N} \mathrm{II}] 6584)$ & $118.7 \pm 0.2$ & $124.0 \pm 1.0$ \\
$E W([\mathrm{~S} \mathrm{II}] 6717,6731)$ & $3.7 \pm 0.3$ & $1 \pm 1$ \\
\hline
\end{tabular}

reason for the absence of indications of nuclear activity in the hard X-rays (Risaliti et al. 2000). In the spectrum from Fig. 6, we do not see clear-cut evidence for an AGN in IRAS 03158+4227; in particular the conventional diagnostic $E W$ ratio [O III] $5007 / \mathrm{H} \beta$ is too low for a Seyfert nucleus. However, the signal-to-noise ratio is low for these lines, and, owing to the low dispersion, we are not able to resolve $\mathrm{H} \alpha+[\mathrm{N} \mathrm{II}] 6584$. The low intensity of the emission lines in the blue part of the spectrum is probably best explained as due to strong internal dust absorption and resultant reddening, though the effect of the underlying stellar absorption is difficult to estimate. If $\mathrm{H} \beta$ is significantly affected by stellar absorption of an older, A-type burst population, the intrinsic [O III] $5007 / \mathrm{H} \beta$ ratio would be even less compatible with a Seyfert spectrum. On the other hand, the diagnostic line ratios of G2 are more consistent with an AGN. It should be noted that the $K^{\prime}$-band image of G1 is more concetrated than that of G2 (Fig. 5), as seems typical for ULIRGs compared to AGN hosts (Sanders et al. 2000).

\section{Discussion}

The long, curved tail of G2 seen in Fig. 3, along with the fact that G1 and G2 have nearly the same redshift, may be taken as an indication for the gravitational interaction of G1 and G2. Such tails of escaping debris from the far side of a victim disk are well-known indicators of the encounter of nearly equal-mass spiral galaxies (e.g. Toomre \& Toomre 1972; Schombert et al. 1990). On the other hand, if the ULIRG activity was triggered by this interaction, the large projected distance between these two galaxies is surprising. Murphy et al. (1996) suggested that ULIRGs with large $(>10 \mathrm{kpc})$ nuclear separation may represent a triple merger with a third, undetected nucleus from a previous encounter or, alternatively, that the ULIRG phenomenon can occur in an early phase of the interaction. Below, we briefly discuss IRAS $03158+5228$ in the light of these two scenarios.

Arguments in favour of the multiple merger scenario were derived from the properties of elliptical galaxies (e.g., Barnes 1984; Mamon 1987; Schweizer 1989; Weil \& Hernquist 1996), from the dynamical diversity of ULIRGs (Borne et al. 2000; Cui et al. 2001), and from detailed studies of individual galaxies (Taniguchi \& Shioya 1998; Lipari et al. 2000). Multiple encounters and mergers 


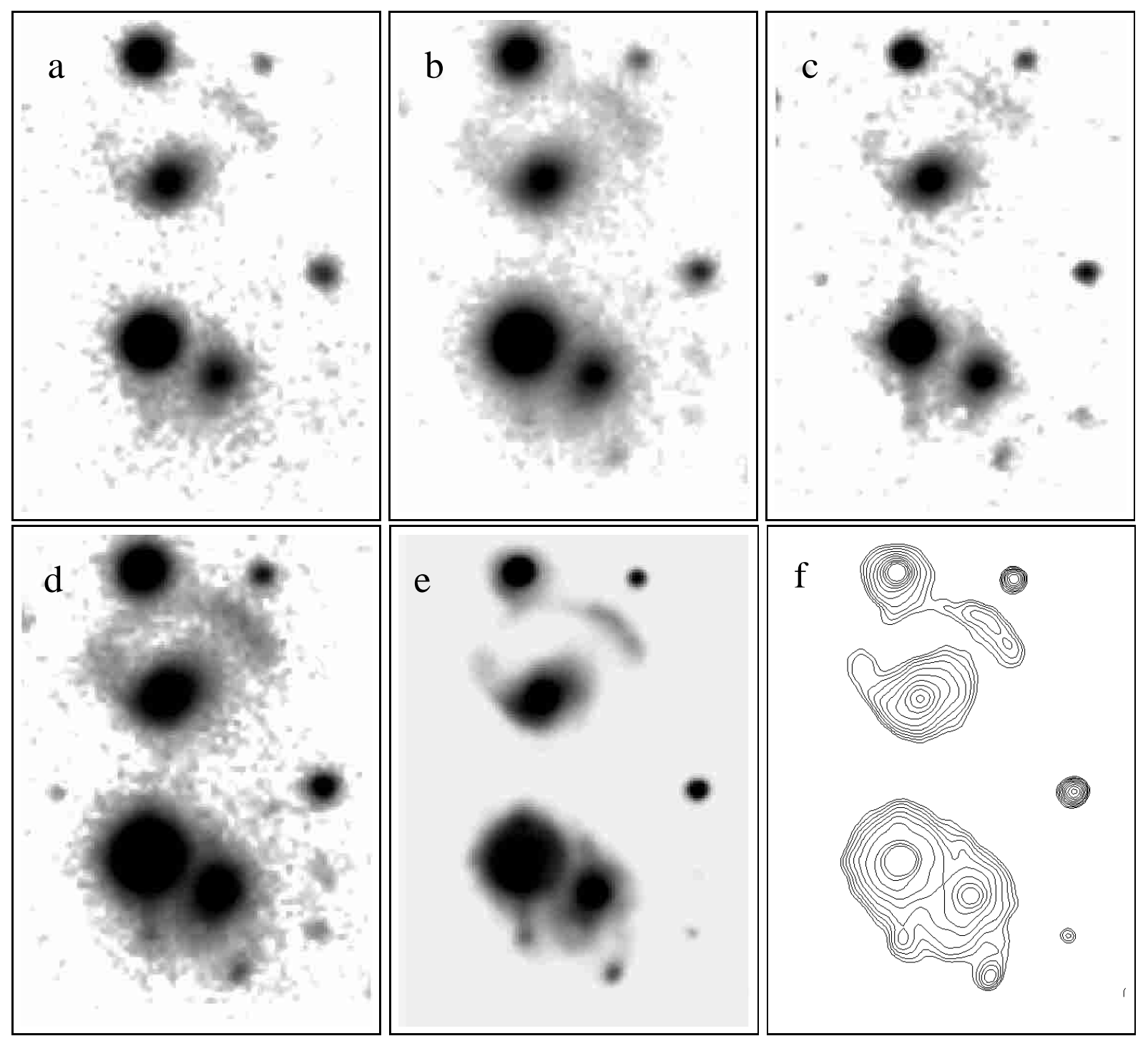

Fig. 3. Optical images of IRAS 03158+4227: a) $B$-band; b) $R$-band; c) $I$-band; d) co-addition of all optical images; e) the same image as $\mathbf{d}$ ), but after Lucy-Richardson deconvolution with 10 iteration steps; $\mathbf{f}$ ) the contour plot of image e) where successive contour lines correspond to a factor 3 in intensity. (The unfiltered image shows the same structures as the images a) to c) and is not displayed here.) The size of each image is $30^{\prime \prime} \times 42^{\prime \prime}, \mathrm{N}$ is up, E is left.

are suggested to occur naturally in compact groups of galaxies (Barnes 1989; Hickson 1997; Borne et al. 2000; Bekki 2001). It seems likely that a fraction of ULIRGs is triggered by such a process. Borne et al. (2000) and Cui et al. (2001) considered the appearance of double or multiple nuclei as a keytest for the multiple merger origin and derived percentages of $20 \%$ and $17 \%$, respectively, of multi-nuclei ULIRGs. The fraction of ULIRGs triggered by multiple mergers is certainly larger than the fraction of multi-nuclei systems, since a multiple nucleus is expected to evolve on a short timescale to a double nucleus and finally to a single nucleus. Unfortunately, this method is faced with serious difficulties which can lead to an overestimation of multi-nuclei systems: the morphology of the central regions of ULIRGs has the tendency to be strongly affected by dust obscuration effects and by the appearance of regions of intense star formation on a scale of kpc or subkpc. Further, the studies mentioned above did not identify real interacting members with spectroscopic observations. Following Bekki (2001), it seems fair to say that the fraction of ULIRGs formed by multiple merging is still highly uncertain.

Dinh-V-Trung et al. (2001) have studied the six systems with nuclear separations larger than $20 \mathrm{kpc}$ among the ULIRGs from the complete 1 Jy sample (Kim \& Sanders 1998). The optical and $K^{\prime}$-band imaging observations and optical spectra suggest the multiple merger scenario for only one of those ULIRGs, IRAS $14394+5332$. It cannot be excluded that IRAS $03158+4227$ is a multiple merger like IRAS $14394+5332$. Indeed, the morphologies 

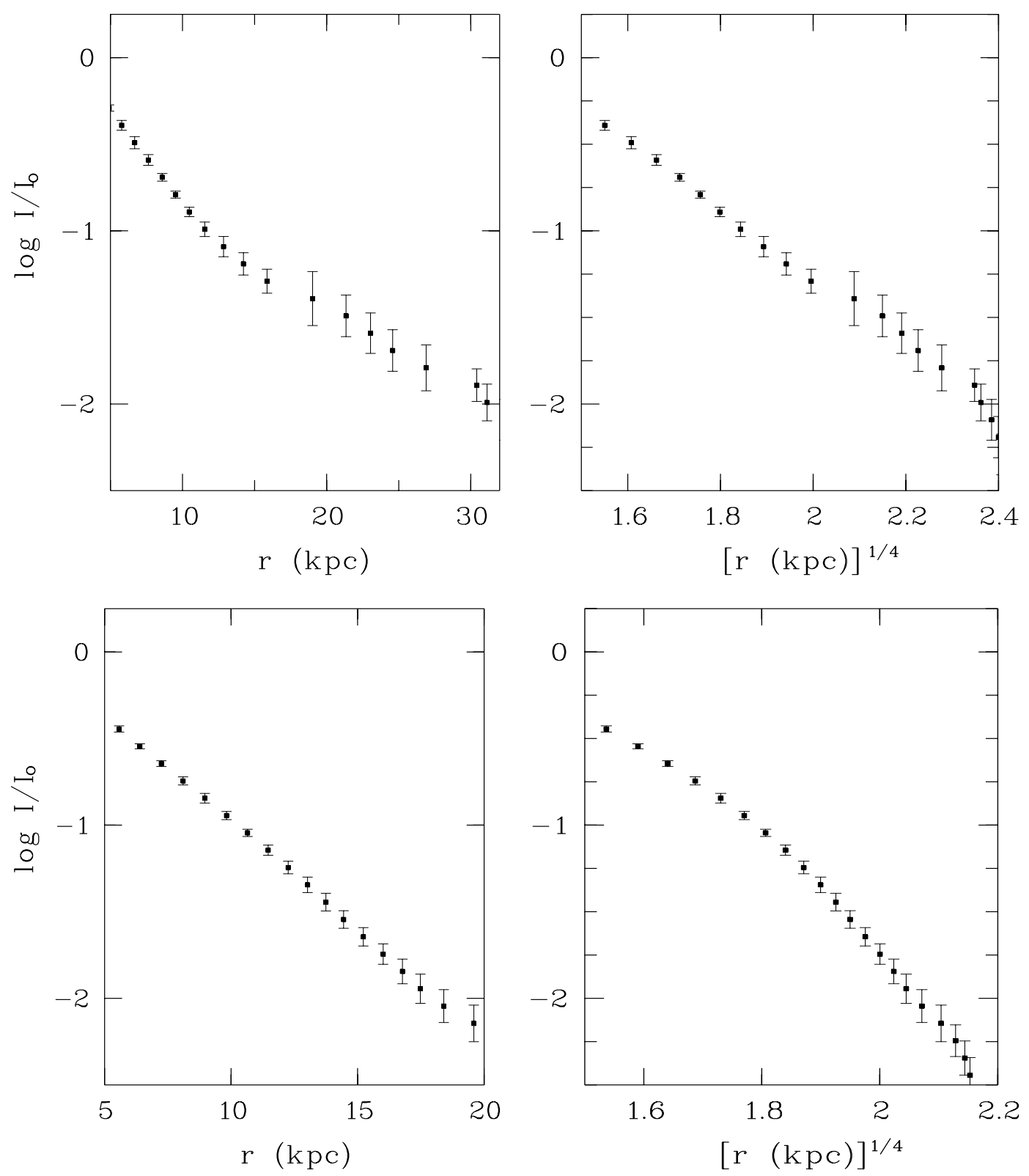

Fig. 4. Intensity profiles of G1 (top) and G2 (bottom) derived from the $R$-band image by means of the MIDAS procedure fit/ell3. $I_{0}$ is the central intensity. The angular interval where the image of G1 is affected by the star S1 was excluded from the profile analysis.

of these two systems show some similarities. Although we do not find evidence for a close double nucleus, IRAS 03158+4227 might be in a more advanced stage, where two nuclei of G1 have coalesced and the inner region is already well relaxed, as seems to be indicated by the radial luminosity profile (Fig. 4).

How can the nuclear activity of G2 be matched by such a scenario? Nuclear activity is not unusual in ULIRGsystems, though the active nuclei are mostly located in the hosts of the ULIRG itself. However, there is one system (IRAS 17028+5817) among the widely separated pairs studied by Dinh-V-Trung et al. where the spectrum of the ULIRG's host is of H II-type whereas the companion has a LINER-type spectrum. We cannot exclude that G2 is also a late merger. However, its disk-like structure admits a variant of a multiple merger where the ULIRG activity was triggered by a past merger and the AGN in G2 by the present interaction between G1 and G2.

The simulations by Bekki (2001) have demonstrated that a multiple merger can trigger repetitive starbursts with a star formation rate comparable to ULIRGs. However, the discussion by Bekki suggests that very intense starbursts with an amplitude of $\gtrsim 10^{2} M_{\odot} / \mathrm{yr}$ are not likely in such an environment. As was stressed already in the Introduction, IRAS $03158+4227$ is one of the most luminous ULIRGs from the 2 Jy sample. According to the relation derived by Clements et al. (1996), the $60 \mu \mathrm{m}$ flux transforms into a high star formation rate of about $2 \times 10^{3} M_{\odot} /$ yr, i.e. much higher than what seems possible in compact groups. 


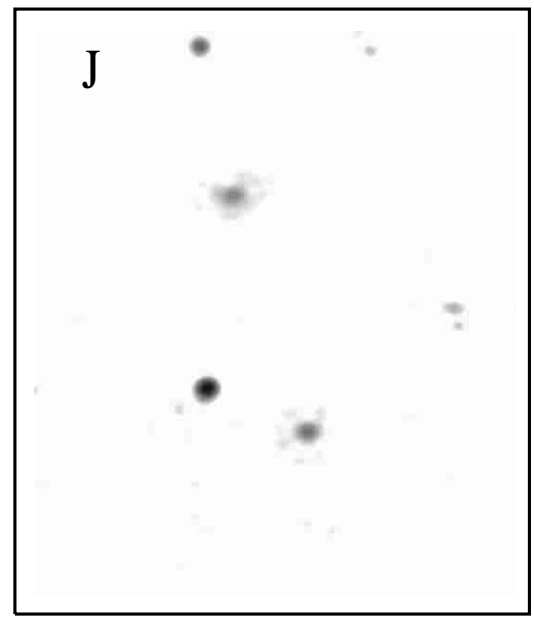

$\mathrm{H}$
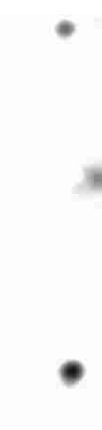

$\mathrm{K}^{\prime}$

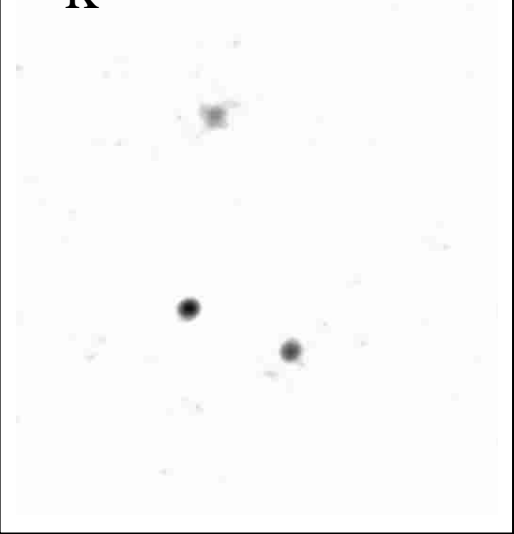

Fig. 5. High-resolution images of IRAS $03158+4228$ in the $J, H$, and $K^{\prime}$ bands. The scale and the size are the same as in Fig. 3 .
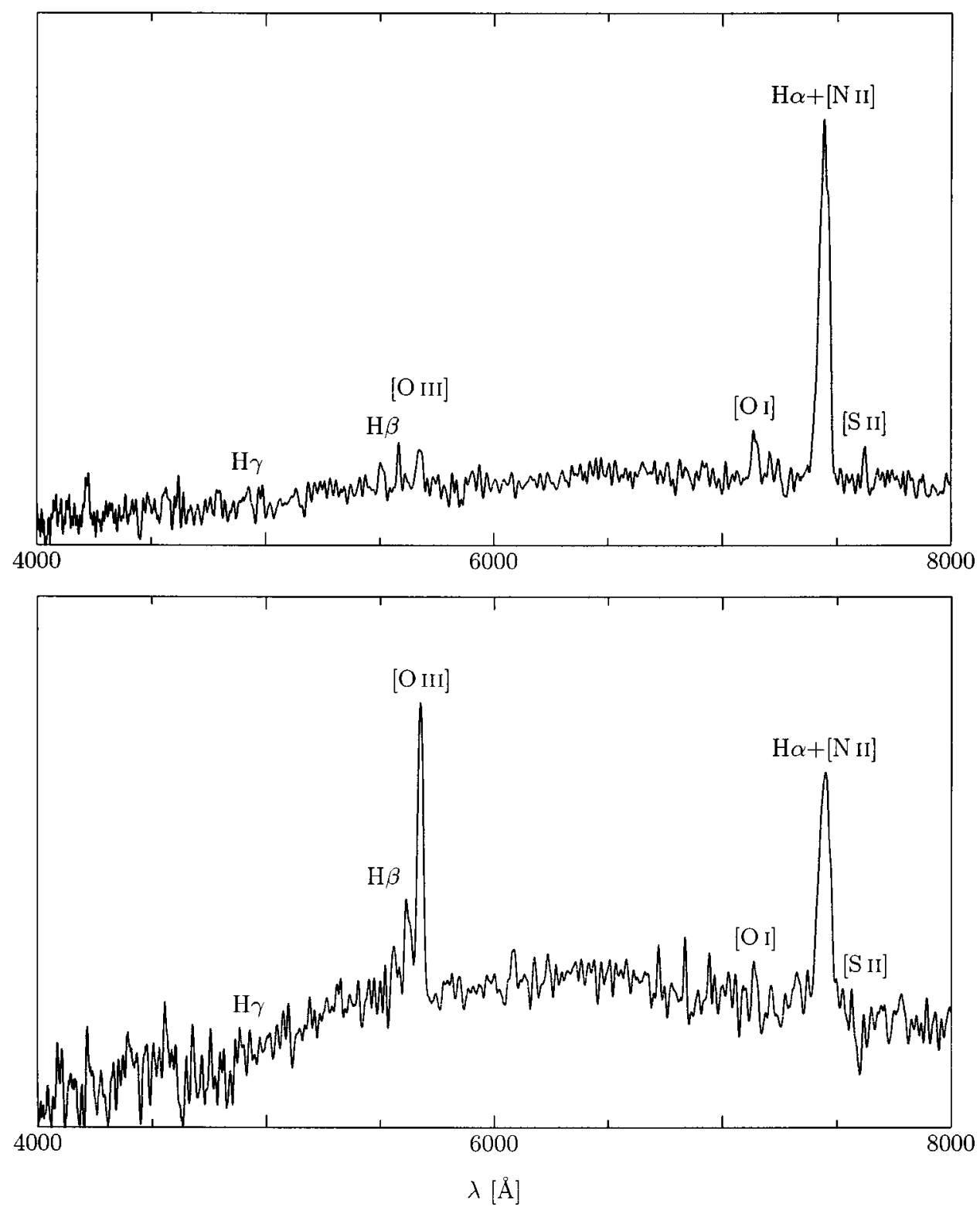

Fig. 6. Optical low-dispersion spectrum (not flux-calibrated, observer frame) of the ULIRG IRAS 03158+4227 (G1, top) and of the galaxy G2 (bottom). 
As an alternative to the multiple merger scenario, it seems tempting to speculate that the activities in the centres of G1 and G2 were triggered by the same process, namely an interaction of G1 and G2. Liu \& Kennicutt (1995, their Fig. 4) discussed the empirical distribution of the equivalent widths of the $\mathrm{H} \alpha+[\mathrm{N}$ II] line for different merger morphological types. The EWs measured for G1 and G2 (Table 2) are in better agreement with Liu \& Kennicutt's morphology type 3 (= systems of two disk galaxies) than with type 2 (= advanced merger which appear to be single). Moreover, according to its infrared colour index $f_{25} / f_{60}<0.2$, IRAS $03158+4228$ belongs to the group of "cool" ULIRGs which are characterized as a major merger with prominent extended tidal structures and resolved double nuclei rather than by small $(<2.5 \mathrm{kpc})$ nuclei separation systems (Surace et al. 2000).

The simulations by Mihos \& Hernquist (1996) have demonstrated that disk/bulge/halo systems with dense central bulges experience strongest gaseous infall and star formation activity in the final stages of coalescence when they are within a few kpc of one another. Their disk/halo models without dense bulges, on the other hand, are most active in earlier phases of merging when the galaxies are separated by tens of kpc. At the beginning of the first starburst phase, the snapshots of the disk/halo merger models by Mihos \& Hernquist (their Figs. 11 and 12) show a remarkable similarity with the few morphological details seen in IRAS 03158+4227: one galaxy (hereafter: g1) is more concentrated, especially the gas and the young stars, with knots and short arms, whereas the most prominent feature of its interaction partner (hereafter: g2) is an extended curved tail at the opposite side. During the next time steps, when the SFR reaches its maximum, the bridge between $\mathrm{g} 1$ and $\mathrm{g} 2$ becomes weaker and $\mathrm{g} 1$ becomes more concentrated.

The long lopsided tail of G2 is the only visible morphologically peculiar feature of the system. It is therefore important for the understanding of the merger stage of IRAS $03158+4227$ to know whether this structure can be due to the tidal interaction with G1. Since it is not possible to follow the evolution of the extended tidal structures in the snapshots shown by Mihos \& Hernquist, we performed a small series of restricted $N$-body simulations like those in Toomre \& Toomre (1972). The main idea of this method is to derive the orbits of both galaxies from the corresponding two-body problem, e.g. by solving a Kepler problem, if the galaxies are treated as point masses. Using these orbits the time-dependent potential at each point is given by a superposition of the two galactic potentials. Stars are treated as test particles, which reduces the classical $N$-body problem to $N$ single-body problems. (Details of the applied code are described in Theis \& Kohle 2001). The main advantages of this method are a fast computation and a high spatial resolution. However, the method is not self-consistent, because effects of selfgravity (like fragmentation or dynamical friction) are neglected. Anyway, comparisons between self-consistent and restricted $N$-body calculations demonstrated in several cases a good agreement, provided the encounters are not too strong and/or the duration of the simulated stage is not very long. Therefore, and because there are not many constraints from observations, the restricted $N$-body simulations should be a good starting point. The results from the present simulations are however considered indicative rather than conclusive.

Figure 7 shows the result of a parabolic encounter with an orbit inclination of $60^{\circ}$, a minimum distance of $15 \mathrm{kpc}$ (reached at $t=0$ ), a line-of-sight-velocity of $200 \mathrm{~km} \mathrm{~s}^{-1}$, and a final projected distance of $50 \mathrm{kpc}$. The total (dynamical) mass of each galaxy is $10^{12} M_{\odot}$. Since we are mainly interested in the tidal shape of G2, we resolved only G2 in test particles. Motivated by the observed radial intensity profile, we distributed the test particles in an exponential disk with a scale length of $5 \mathrm{kpc}$ and a cut-off radius at $15 \mathrm{kpc}$. For simplicity we assumed the disk to be coplanar with the plane of sky. The qualitative agreement between the optical image and the simulations is very good: after $7.5 \times 10^{7} \mathrm{yr}$ a lopsided structure has been formed. This gives rise to an arc-like structure northwest of G2 and a small spur emanating north-east from the main body of G2. In the simulations, these features are the dense parts of a tidal tail which seems also to exist in the observations (e.g. lower-right panel of Fig. 3). The bridge connecting G1 and G2 may be too weak to be clearly detected. Increasing the line-of-sight velocity to $300 \mathrm{~km} \mathrm{~s}^{-1}$ yields very similar results.

The assumption that IRAS $03158+4227$ is triggered by the interaction between G1 and G2 implicates that the ULIRG phenomenon is not restricted to late binary merger stages. Such an interpretation is supported by further indications. Rigopoulou et al. (1999) reported a lack of any correlation between the stage of merger, measured by the separation of nuclei, and the infrared luminosity in an unbiased sample of 62 ULIRGs. Further, there is no trend of increased ULIRG activity in systems with more centrally concentrated $\mathrm{H} \alpha$ emission (Mihos \& Bothun 1998), and also the total mass of molecular gas in ULIRGs is not related to the linear separation (Gao \& Solomon 1999; Rigopoulou et al. 1999). Finally, Dinh-V-Trung et al. (2001) present evidence for IRAS $23327+2913$ to be hosted by a non-disturbed spiral-like galaxy which may be interpreted as an early stage of merging.

\section{Conclusions}

Deep optical images, spectra and high-resolution NIR images are presented for IRAS $03158+4227$, one of the most luminous ULIRGs of the $2 \mathrm{Jy}$ sample. The host galaxy, G1, is identified with a component of a binary of nearly equal-luminosity giant galaxies separated by $47 h_{75}^{-1} \mathrm{kpc}$. The companion galaxy, G2, harbours an active nucleus and has an extended, curved tail. This tail may be interpreted as a tidal structure induced by the gravitational interaction with the host galaxy of the ULIRG. The highresolution NIR images do not reveal any sign for a close 

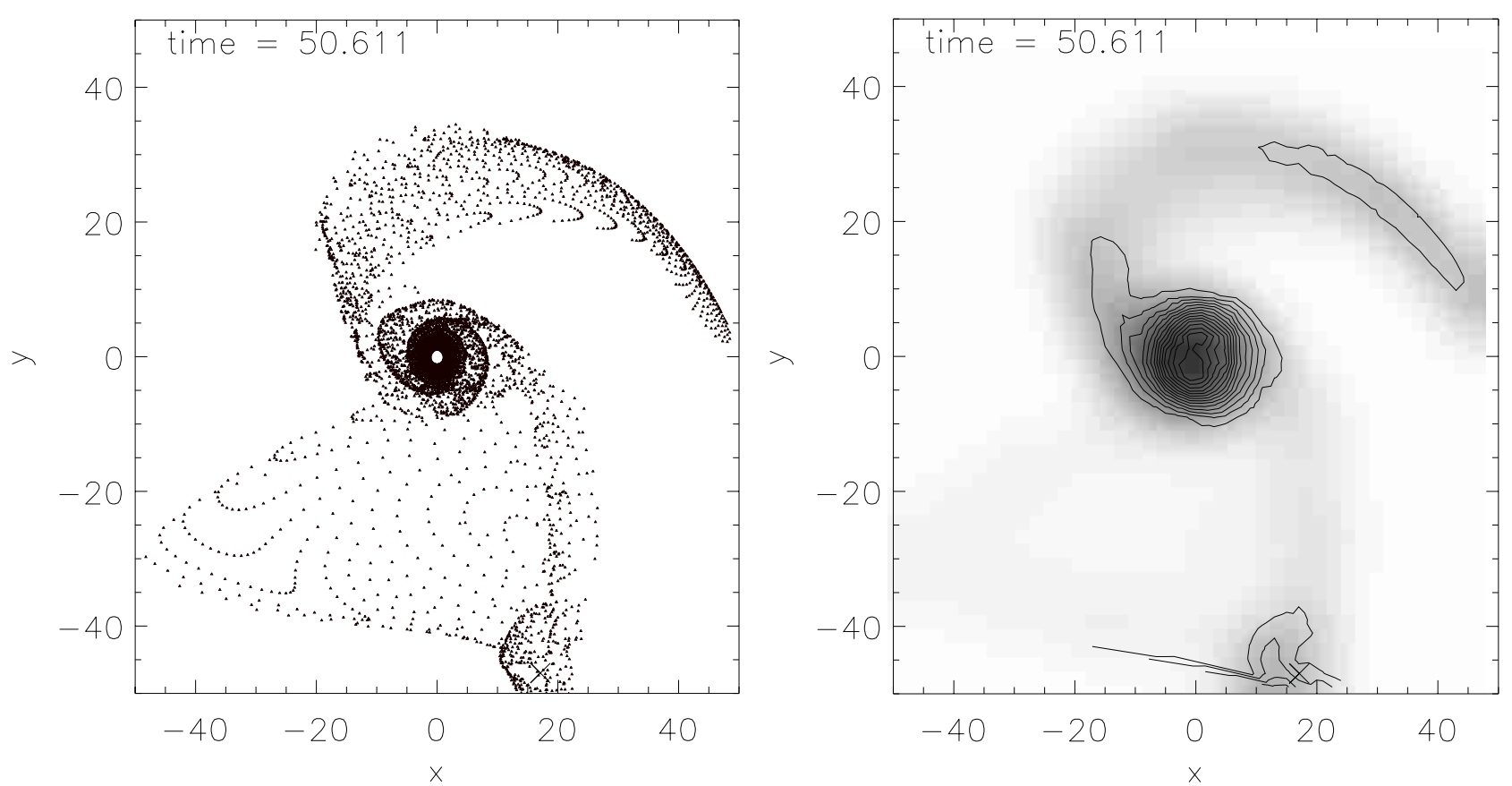

Fig. 7. Simulation of the gravitational perturbation of the galaxy G2 by the galaxy G1. Both galaxies have the same dynamical mass of $10^{12} M_{\odot}$. Left: projected distribution of the mass particles from G2. G1 is marked by the cross at the bottom. The length is measured in $\mathrm{kpc}$ and the time unit is $1.49 \mathrm{Myr}$. No test particles have been used inside the central kpc. Right: blurred intensity plot of the image from the left hand side, overlaid by a contour plot; the lowest intensity contour approximately corresponds to the detection threshold in the optical images of IRAS $03158+4227$.

double nucleus down to a (resolution-limited) nuclei separation of $1 \mathrm{kpc}$.

ULIRGs in such widely separated systems may be explained by a multiple merger, like IRAS $14394+532$ (Dinh-V-Trung et al. 2001), where the interaction of two components has already reached an advanced stage with a very small separation of nuclei. Indeed, the optical luminosity profile of G1 is reasonably fit by a de Vaucouleurs law. The profile of G2 is more compatible with a disklike structure. Hence it seems likely, in the multi-merger scenario, that the ULIRG in G1 was triggered by a past merger and the AGN in G2 by the present interaction between G1 and G2. If the nuclear activity of G2 is also due to a (late) merger, the system IRAS $03158+4227$ would represent the case of the gravitational interaction of two advanced mergers. Such scenarios would imply that multiple mergers in compact groups can trigger very intense starbursts.

As an alternative explanation, both the ULIRG IRAS $03158+4227$ and the nuclear activity in its neighbour galaxy might be dynamically triggered by the same process, namely the gravitational interaction of these two galaxies. In this case, IRAS $03158+4227$ would represent an early stage of binary tidal interaction, and the interpretation of ULIRGs as final merger stages may need to be reexamined.

Acknowledgements. This research is based on observations made with the $3.5 \mathrm{~m}$ telescope and the $2.2 \mathrm{~m}$ telescope of the German-Spanish Astronomical Centre, Calar Alto,
Spain. We acknowledge financial support from the Deutsche Forschungsgemeinschaft under grants Me 1350/3, 5, 8, 14 and Ste 605/15. This research has made use of the NASA/IPAC Extragalactic Database (NED) which is operated by the Jet Propulsion Laboratory, California Institute of Technology, under contract with the National Aeronautics and Space Administration.

\section{References}

Arp, H. C. 1966, ApJS, 14, 1

Arp, H. C., \& Madore, B. F. 1987, A Catalogue of Southern Peculiar Galaxies and Associations (Univ. Press, Cambridge)

Aurière, M., Hecquet, J., Coupinot, G., Arthaud, R., \& Mirabel, I. F. 1996, A\&A, 312, 387

Barnes, J. E. 1984, MNRAS, 208, 873

Barnes, J. E. 1989, Nature, 338, 123

Barnes, J. E., \& Hernquist, L. 1991, ApJ, 370, L65

Barnes, J. E., \& Hernquist, L. 1992, ARA\&A, 30, 705

Barnes, J. E., \& Hernquist, L. 1996, ApJ, 471, 115

Bekki, K. 2001, ApJ, 546, 189

Bekki, K., \& Noguchi, M. 1994, A\&A, 290, 7

Borne, K. D., Bushouse, H., Lucas, R. A., et al. 2000, ApJ, 529, L77

Brunzendorf, J., \& Meusinger, H. 1999, A\&AS, 139, 141

Clements, D. L., Sutherland, W. J., Saunders, W., et al. 1996, MNRAS, 279, 459

Condon, J. J., Cotton, W. D., Greisen, E. W., et al. 1998, AJ, 115,1693

Cui, J., Xia, X.-Y., Deng, Z.-G., et al. 2001, AJ, 122, 63 
Cutrie, R. M., Skrutskie, M. F., Van Dyck, S., et al. 2000 http://www.ipac.caltech.edu/2mass/releases/second/ doc/explsup.html

Coleman, G. D., Wu, C.-C., \& Weedman, D. W. 1980, ApJS, 43, 393

Dinh-V-Trung, Lo K. Y., Kim, D.-C., et al. 2001, ApJ, 556, 141

Duc, P.-A., Mirabel, I. F., \& Maza, J. 1997, A\&AS, 124, 533

Gao, Y., \& Solomon, P. M. 1999, ApJ, 512, L99

Genzel, R., Lutz, D., Sturm, E., et al. 1998, ApJ, 498, 579

Helou, G., Soifer, B. T., \& Rowan-Robinson, M. 1985, ApJ, 298, L7

Hickson, P. 1997, ARA\&A, 35, 357

Hippler, S., Glindemann, A., Kasper, et al. 1998, Proc. SPIE, 3353, 44

Joseph, R. D., \& Wright, G. S. 1985 MNRAS, 214, 87

Kennicutt, R. C. 1996, in Galaxies: Interactions and Induced Star Formation, ed. R. C. Kennicutt, F. Schweizer, \& J. E. Barnes (Springer, Berlin), 1

Kim, D.-C., \& Sanders, D. B. 1998, ApJS, 119, 41

Lípari, S., Díaz, R., Taniguchi, Y., et al. 2000, AJ, 120, 645

Liu, C. T., \& Kennicutt, R. C. 1995, ApJ, 450, 547

Mamon, G. A. 1987, ApJ, 321, 622

Mihos, J. C., \& Hernquist, L. 1996, ApJ, 464, 641

Mihos, J. C., Dubinski, J., \& Hernquist, L. 1998, ApJ, 494, 183

Mihos, J. C., \& Bothun, G. D. 1998, ApJ, 500, 619

Monet, D., Bird, A., Canzian, B., et al. 1998, A Catalog of Astrometric Standards, U.S. Naval Obs. Flagstaff Station and Universities Space Res., Assoc.

Murphy, T. W., Armus, L., Matthews, K., et al. 1996, AJ, 111, 1025
Negroponte, J., \& White, S. D. M. 1983, MNRAS, 205, 1009

Noguchi, M. 1991, MNRAS, 251, 360

Noguchi, M., \& Ishibashi, S. 1986, MNRAS, 219, 657

Pantin, E., \& Starck, J. L. 1996, A\&AS, 118, 575

Rigopoulou, D., Spoon, H. W. W., Genzel, R., et al. 1999, AJ, 118,2625

Rieke, G. H., \& Low, F. J. 1972, ApJ, 176, L95

Risaliti, G., Gilli, R., Maiolino, R., et al. 2000, A\&A, 357, 13

Sanders, D. B., Soifer, B. T., Elias, J. H., et al. 1988, ApJ, 325,74

Sanders, D. B., \& Mirabel, I. F. 1996, ARA\&A, 34, 749

Sanders, D. B., Kim, D.-C., Mazzarella, J. M., et al. 2000, in Galaxy Dynamics: from the Early Universe to the Present, ed. F. Combes, G. A. Mamon, \& V. Charmandaris, ASP Conf. Ser., 197, 295

Schombert, J. M., Wallin, J. F., \& Struck-Marcell, C. 1990, AJ, 99, 497

Schweizer, F. 1989, Nature, 338, 119

Scoville, N. Z., Evans, A. S., Thompson, R., et al. 2000, ApJ, 119, 991

Soifer, B. T., Houck, J. R., \& Neugebauer, G. 1987, ARA\&A, 25,187

Spoke, C. 1997, ApJS, 113, 269

Surace, J. A., Sanders, D. B., \& Evans, A. S. 2000, ApJ, 529, 170

Taniguchi, Y., \& Shioya, Y. 1989, ApJ, 501, L167

Theis, Ch., \& Kohle, S. 2001, A\&A, 370, 365

Toomre, A., \& Toomre, J. 1972, ApJ, 178, 623

Weil, M. L., \& Hernquist, L. 1996, ApJ, 460, 101 